\title{
THE INFLUENCE OF TOTAL BASE INK COVERAGE ON THE UNIFORMITY OF DIGITAL PRINTS OVERPRINTED WITH PEARLESCENT INKS
}

\author{
Ivana Tomić (iD), Ivan Pinćjer (D), Nada Miketić \\ University of Novi Sad, Faculty of Technical Sciences, \\ Department of Graphic Engineering and Design, Novi Sad, Serbia
}

\begin{abstract}
Print uniformity is an important parameter that can determine perceived quality of a printed product. If the product is of low print uniformity, its quality is often regarded as non-satisfactory. In this work we were interested in the uniformity of electrophotography prints overprinted with inks containing pearlescent pigments. Our goal was to determine whether the overall print uniformity was influenced by the total base ink coverage i.e. the ink coverage of the printing substrate before pigments were applied to it. Hence, three scenarios were considered: when pearlescent inks were printed over the unprinted paper, previously printed grey, and black colour patch. Nine different types of pearlescent pigments, dispersed in a transparent ink vehicle, were screen printed over the paper and the previously printed patches of grey and black colour. The base colours were printed in electrophotography. The uniformity of prints obtained in such a manner was determined by calculating GLCM parameters that were shown to correlate well with human perception of uniformity. It was shown that overprinting the electrophotography prints with pearlescent inks significantly decreased the uniformity of prints with black base colour and slightly improved the uniformity of those with grey colour. When pearlescent inks were printed over the paper, the uniformity did not change significantly. Observing the print uniformity of overprinted samples, the best results were obtained when pearlescent inks were printed directly to the paper, following the cases when they were printed over the grey and black prints. It is therefore concluded that the total base ink coverage has significant effect on the uniformity of electrophotography prints overprinted with pearlescent inks. The results indicate poor adhesion of the ink vehicle used as a carrier for pearlescent pigments to electrophotographic toner.
\end{abstract}

Key words: print uniformity, electrophotography, pearlescent pigments

\section{INTRODUCTION}

Together with colour and sharpness, print uniformity is one of the most important parameter influencing perception of a printed product (Jurič, 2018). If there is a variation in density in the areas that are expected to be uniform (areas of one colour) the print is perceived as non-uniform and is rated as being of lower quality. The non-uniformity can be micro and macro (ISO/IEC, 2017), while the variations in density can be random or systematic (Christoffersson, 2002).

The non-uniformity depends on the printing process, paper and the ink used, as well as their interaction (Jurič, 2018; Sadovnikov et al, 2005). It is often a problem in electrophotography, due to the nature of the printing process (Jurič, 2018; Sipi, 2002). In this work we were interested whether the non-uniformity of electrophotography prints can be improved by overprinting, where we considered using inks with pearlescent pigments. These types of pigments are often used in packaging for achieving the effect of subtle, pearl shine, as well as the colour travel effect (change of a colour with the change of the angle of viewing/illuminating). Pearlescent pigments are described by their interference colour, which is a colour observed near the specular angle of reflection (Klein, 2010). They consist of a substrate which is almost always natural or synthetic muscovite - mica, and the layers of different materials. It is their structure that enables abovementioned optical effects, and determine the size and the shape of pigment flakes (which further influence the uniformity of prints if those pigments are used in printing inks).

In this work we place a focus on the interaction of electrophotography ink and pearlescent inks. The goal was to test whether print uniformity of overprinted samples depends on the amount of the electrophotography toner on the printing surface. So, three options were considered - pearlescent inks were printed over the unprinted paper, as well as the previously printed grey and black ink. In this manner we tested how the electrophotography ink layer interacts with pearlescent ink and whether the uniformity of overprinted sample is actually influenced by the total base ink coverage. 


\section{METHOD}

For this study we used nine pearlescent pigments that differed in their composition and, therefore, the interference colour. Almost all the pigments have muscovite mica as a carrier and a different number of additional layers (Table 1). For brevity, in the rest of the paper we will name the pigments by their interference colours.

Table 1: Pigments used in the study

\begin{tabular}{|l|l|c|l|}
\hline Pigment & $\begin{array}{l}\text { Interference } \\
\text { colour (name) }\end{array}$ & $\begin{array}{l}\text { Number of } \\
\text { components }\end{array}$ & Composition \\
\hline 504 Red & Red & 2 & Mica coated with: ferric oxide \\
\hline 221 Rutile Fine Blue & Pearl-gold & 3 & $\begin{array}{l}\text { Mica coated with: titanium dioxide, ferric } \\
\text { oxide }\end{array}$ \\
\hline 231 Rutile Fine Green & Green & 3 & $\begin{array}{l}\text { Mica coated with: titanium dioxide, tin } \\
\text { oxide }\end{array}$ \\
\hline 223 Rutile Fine Lilac & Lilac & $\begin{array}{l}\text { Mica coated with: titanium dioxide, tin } \\
\text { oxide }\end{array}$ \\
\hline 325 Solar gold satin & Gold & 3 & $\begin{array}{l}\text { Mica coated with: titanium dioxide, tin } \\
\text { oxide }\end{array}$ \\
\hline Blue-shade silver SW 9605 & Blue-silver & 4 & $\begin{array}{l}\text { Mica coated with: titanium dioxide, ferric } \\
\text { oxide, silicon dioxide }\end{array}$ \\
\hline T20-03 WNT Tropic sunrise & $\begin{array}{l}\text { Green- } \\
\text { orange* }\end{array}$ & 5 & $\begin{array}{l}\text { Silicon dioxide coated with: titanium } \\
\text { dioxide, tin oxide, zirconium oxide, } \\
\text { auxiliaries }\end{array}$ \\
\hline GP Rutile Blue Green WNT & Turquoise & 7 & $\begin{array}{l}\text { Mica coated with: titanium dioxide, cobalt } \\
\text { titanate, zirconium oxide, aluminium oxide, } \\
\text { tin oxide, auxiliaries }\end{array}$ \\
\hline
\end{tabular}

*Interference colour for the selected pigment is green, but it is named differently in order not to be confused with the 231 Rutile Fine Green. Orange was chosen since the pigment provides green through silver, red and orange colour flop

As a printing substrate we used matte art paper grammage of $300 \mathrm{~g} / \mathrm{m} 2$. The grey and black patches ( $\mathrm{K}$ of $50 \%$ and $100 \%$ ) size of $10 \times 10 \mathrm{~cm}$ were printed on Xerox DocuColor 252 digital printer. After printing and drying they were overprinted with the pearlescent pigments dispersed in the aqueous ink vehicle. Pigments' concentration in the vehicle was 15\%. Overprinting was done in screen printing in order to increase the thickness of the pearlescent inks' layer. We used screen with the mesh count of $43 \mathrm{l} / \mathrm{cm}$. Two layers were deposited over the paper and previously printed patches, after which the prints were dried in the temperature of $140^{\circ}$ during 1 minute.

Prints were further digitized by Canon CanoScan $5600 \mathrm{~F}$ scanner. All the samples (paper, prints without and with pearlescent inks' layer) were scanned in the resolution of $600 \mathrm{ppi}$. Prior scanning, all the automatic options for colour and image correction were turned off in order not to change the initial data. Scanned images were saved as non-tagged TIFF files. The area of $2000 \times 2000$ px of the scanned images were used for subsequent processing.

To determine print uniformity, we used Haralik's method based on the second order statistical measurements through the Grey-Level Co-occurrence Matrix (GLCM) (Gebeješ et al, 2012; Jurič et al, 2016). Of all the parameters that can be obtained from GLCM we choose two that were shown to correlate well with human perception of uniformity - Homogeneity and Entropy (Gebeješ et al, 2013; Gebeješ et al, 2016). Homogeneity measures the uniformity of the non-zero entries in GLCM (Clausi, 2002) and it ranges from 0-1, where 1 indicates completely uniform surface. Entropy is a measure of spatial disorder (Gebeješ et al, 2012), where entropy value of 0 indicates solid colour image. Higher entropy would mean more random distribution of the pixel values.

Calculation of the parameters was performed in Matlab R2016a. All the parameters were calculated from the lightness channel ( $L^{*}$ in CIELAB colour space). We used the distance of $5 \mathrm{px}$ (Tomić, 2016) and the calculation was performed in all directions (horizontal, vertical, $-45^{\circ}$ and $45^{\circ}$ ). The mean value of the parameter calculated in all four directions was taken into account for further analysis. 


\section{RESULTS}

Homogeneity and Entropy values for samples where pearlescent inks are printed over the paper are shown in Figure 1. As a reference, each graph contains the corresponding parameters for unprinted paper.

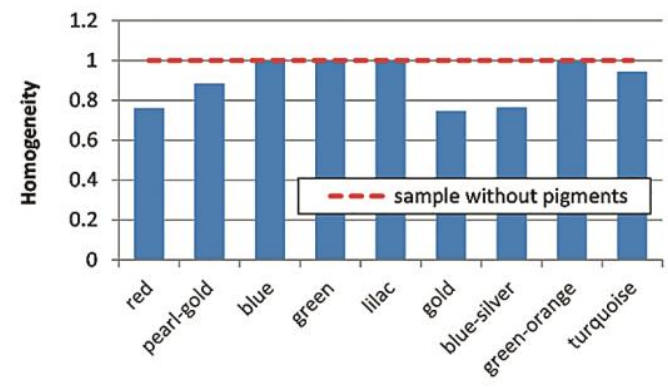

Pigments used for overprinting

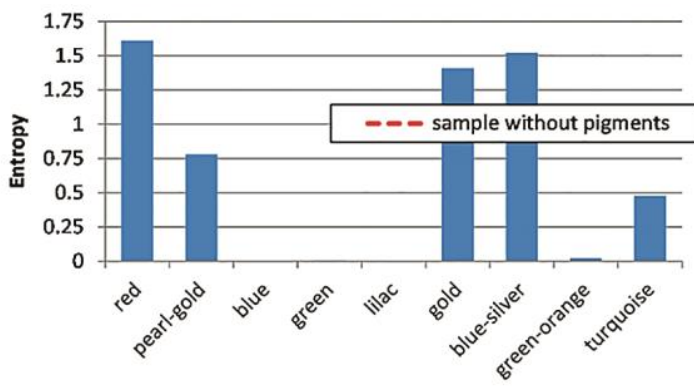

Pigments used for overprinting

Figure 1: Homogeneity and Entropy values of paper printed with pearlescent inks

Unprinted paper used in the experiment had the most uniform surface (Homogeneity was 1, while the Entropy was 0). Adding the pearlescent inks did not change the uniformity in case of ink with Blue, Green, Lilac and Green-Orange pigment - Homogeneity of those prints was very close to 1, while Entropy was quite close to $0(0.005,0.006,0.004$ and 0.025 , respectively) and hence could not be represented well in Figure $1 \mathrm{~b}$. Since all the above-mentioned pigments are (semi)transparent the obtained result can be explained by their optical properties. Namely, pearlescent pigments often consists of more than two layers of different materials. Different refraction indexes of those layers leads to constructive interference, which allows the change of the perceived colour with the change of the angle of light incidence or viewing. When (semi)transparent pigments are printed over the white background, the light of complementary colour is transmitted through the pigment and reflected back in the specular angle. That means that the colour detected in the specular angle is the combination of the interference colour and its complementary colour i.e. white. During scanning the detector was positioned in the specular angle, so what it detected was white, uniform colour.

The rest of the pigments are not transparent, hence the reflected light has different colour. In these cases the uniformity decreased. The smallest change were seen for Turquoise pigment. This pigment dissolved very well in the vehicle, so its spread over the paper was better in comparison to the rest of the pigments used.

Figure 2 shows the Homogeneity and Entropy values for prints where pearlescent inks were printed over grey colour.

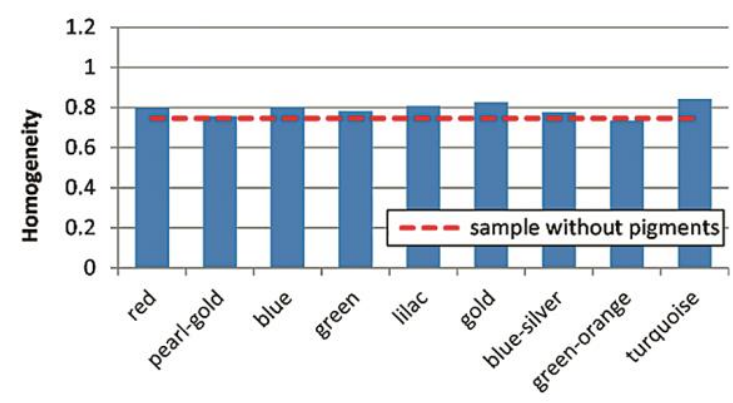

Pigments used for overprinting

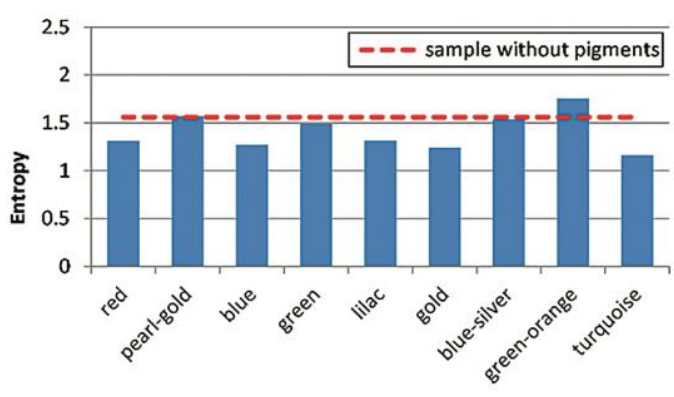

Pigments used for overprinting

Figure 2: Homogeneity and Entropy of prints with grey base colour

Grey patch printed in electrophotography had lower Homogeneity and higher Entropy in comparison to the unprinted paper. This means that it was less uniform than both white and black (see Figures 1-3) colour. In electrophotography the reasons for non-uniformity are mostly related to the process of the printing (Sipi, 2002) where random variations are dominant. 
When pearlescent inks were printed over the grey patch the Homogeneity was the same or even better for all the pigments. Furthermore, the uniformity expressed through this parameter seems not to change much regardless of the pigment used. Entropy was only higher in the case of Green-Orange pigment, while in the rest of the cases it slightly decreased or stayed unchanged. This means that overprinting the grey patch with pearlescent inks slightly increased its print uniformity, lowering the errors caused by the printing process.

Homogeneity and Entropy values for black samples overprinted with pearlescent inks are shown in Figure 3.
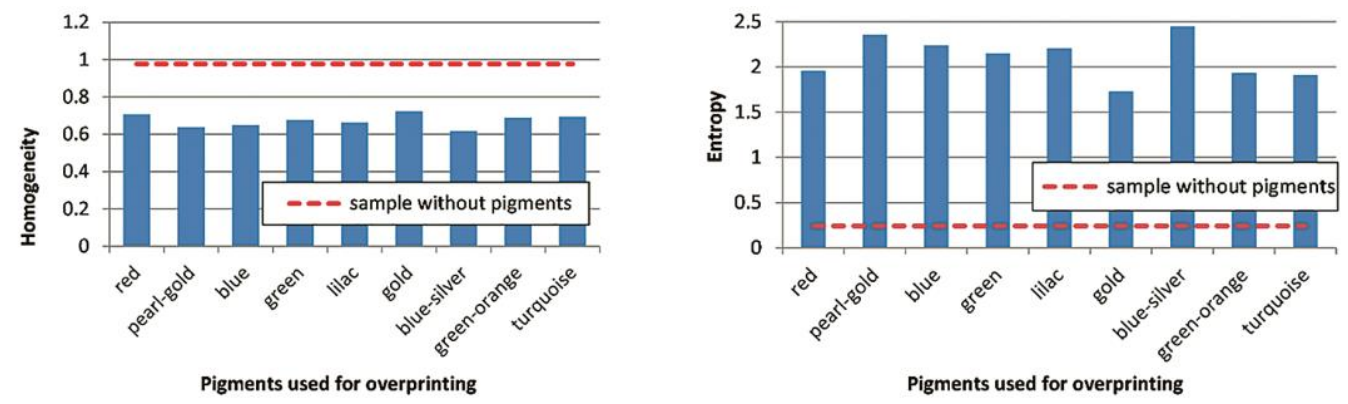

Figure 3. Homogeneity and Entropy of prints with black base colour

Black patch had almost perfect uniformity with Homogeneity quite close to 1 and Entropy of just 0.24. This is understandable since black ink is completely covering the paper. Adding the pearlescent inks in this case reduced uniformity which can be seen in lower Homogeneity and drastic increase in Entropy (Figure 3). By observing the scanned images it was noticed that in all the cases parts of the prints were uncovered by pigments (holes in the pearlescent pigment layer can be clearly seen in images on the left, Figure 4). This was not the case when pigments were applied over the grey colour, where pearlescent ink penetrates to the parts of the patch that was not covered by the toner (see Figure 4, images on the right). Lower uniformity of black patch may indicate poor interaction of aqueous ink vehicle and the electrophotography toner.

(a)

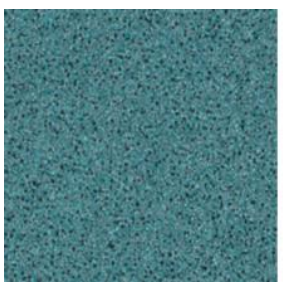

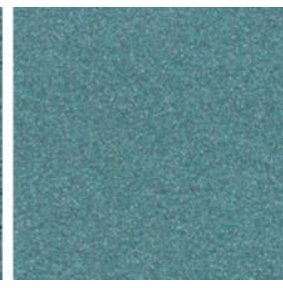

(b)
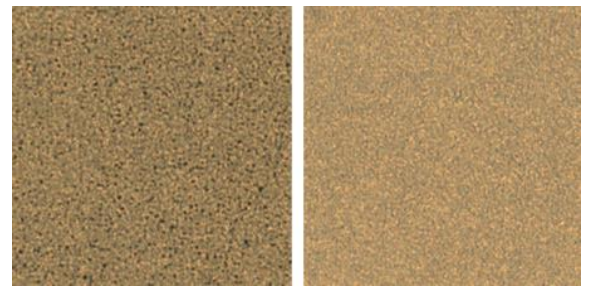

Figure 4. Part of the scanned image of (a) black and grey patch overprinted with Blue-silver pigment, (b) black and grey patch overprinted with Pearl-gold pigment

The Homogeneity and Entropy of overprinted samples with respect to the base colour are shown in Figure 5 and Figure 6.

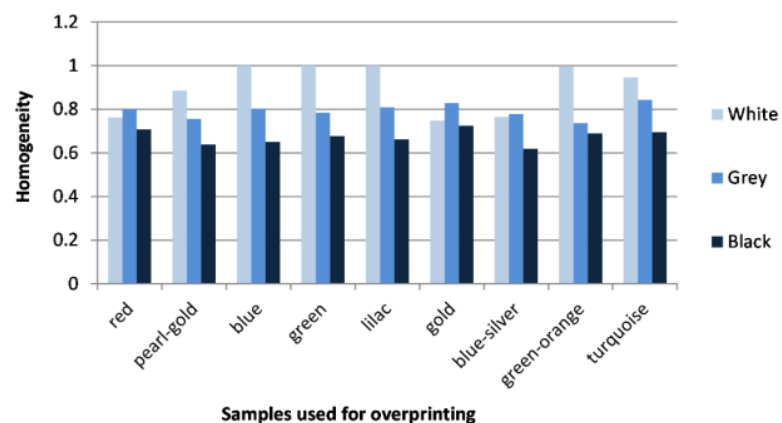

Figure 5. Comparative view of print Homogeneity with respect to the base colour 


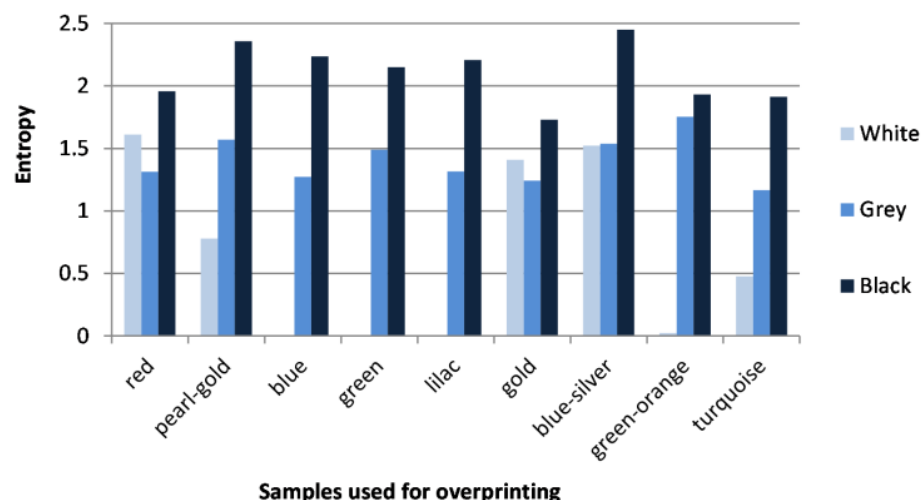

Figure 6. Comparative view of print Entropy with respect to the base colour

From the comparative view it is evident that print uniformity was the best when pigments were printed directly to the paper (in almost all the cases Homogeneity was the highest and Entropy lowest), then grey and finally black ink. Gold and Red pigment are the only two exceptions where samples with grey base colour has slightly better uniformity in comparison to white.

\section{CONCLUSIONS}

Pearlescent pigments are used in printing in order to achieve special visual effects, such as the effect of a pearl shine and colour travel effect. In this work we evaluated print uniformity of the electrophotography prints overprinted with inks containing pearlescent pigments. The goal was to determine the interaction of the electrophotography toner and the pearlescent pigments and to assess how the overall print uniformity is influenced by the amount of the base ink in the printing surface.

The results showed that the uniformity of electrophotography prints without pearlescent pigments was the highest for black and white base colour. The grey patch was quite non-uniform which can be attributed to the nature of the printing process. Adding the pearlescent pigments' layer significantly decreased the uniformity of prints with black base colour and slightly improved the uniformity of grey prints. When pigments were printed over the paper, the uniformity did not change in five cases, while in the rest four it was somewhat lower.

The best results in print uniformity of overprinted samples were obtained when pigments were printed directly to the paper, following the cases when they were printed over the grey and black prints. Therefore, it is concluded that the total base ink coverage significantly influences the uniformity of electrophotography prints overprinted with pearlescent inks. Such results can be explained by the lower capacity of electrophotography toner to attract aqueous ink vehicle in which pearlescent pigments were dispersed. Hence, if the pearlescent pigments are to be printed over the solid colour patch printed in electrophotography alternative options for ink carriers should be considered.

\section{ACKNOWLEDGMENTS}

This research (paper) has been supported by the Ministry of Education, Science and Technological Development through the project no. 451-03-68/2020-14/200156: "Innovative scientific and artistic research from the FTS (activity) domain".

\section{REFERENCES}

[1] Clausi, D.-A.: "An analysis of co-occurrence texture statistics as a function of grey level quantization", Canadian Journal of Remote Sensing, 28 (1), 45-62, 2002. doi: 10.5589/m02-004

[2] Christoffersson, J.: "Evaluation of Systematic \& Colour Print Mottle", MSc thesis, Linkoping University, 2002.

[3] Gebeješ, A., Tomić, I., Huertas, R., Stepanić, M.: “A preliminary perceptual scale for texture feature parameters", 6. International Symposium on Graphic Engineering and Design, (Faculty of Technical Sciences: Novi Sad, Serbia, 2012), pages 195-201. 
[4] Gebeješ, A., Huertas, R., Tomić, I., Stepanić, M.: "Selection of optimal features for texture characterization and perception", 1. Colour and Visual Computing Symposium - CVCS, (The Colour and Visual Computing Laboratory at NTNU: Gjovik, Norway, 2013), pages 1-5. doi: 10.1109/CVCS.2013.6626278

[5] Gebeješ, A., Huertas, R., Tremeau, A., Tomić, I., Biswas, P., Fraza, C., Hauta-Kasari, M.: "Texture Characterization by Grey-Level Co-occurrence Matrix from a Perceptual Approach", 24th Color Imaging Conference - CIC24, (Society for Imaging Science and Technology: San Diego, USA, 2016), pages 271-277. doi: 10.2352/ISSN.2169-2629.2017.32.271

[6] ISO/IEC: "ISO/IEC 24790:2017 - Information technology - Office equipment - Measurement of image quality attributes for hardcopy output - Monochrome text and graphic images", URL: https://www.iso.org/standard/69796.html (last request: 2020-08-05), 2017.

[7] Jurič, I., Karlović, I., Novaković, D., Tomić, I.: "Comparative study of different methods for the assessment of print mottle", Color Research and Application 41 (5), pages 493-499, 2016. doi: 10.1002/col.21984

[8] Jurič, I. "Print uniformity model for digital prints", PhD thesis, University of Novi Sad, 2018.

[9] Klein, G. A.: "Industrial Color Physics", (Springer, London, 2010.)

[10] Sadovnikov, A., Salmela, P., Lensu, L., Kamarainen, J-K., Kälviäinen, H.: "Mottling Assessment of Solid Printed Areas and Its Correlation to Perceived Uniformity", 14th Scandinavian Conference on Image Analysis, (SCIA: Joensuu, Finland, 2005), pages 409-418.

[11] Sipi, K.: "Toner-paper interactions induced by the fixing process in electrophotographic printing", Licentiate's thesis, Helsinki University of Technology, 2002.

[12] Tomić, I., Dedijer, S., Jurič, I., Pál, M.: "The Effect of Scanning Resolution and Displacement Value on the GLCM-Based Features for Paper Texture Characterization", 8. International Symposium on Graphic Engineering and Design, (Faculty of Technical Sciences: Novi Sad, Serbia, 2016), pages 263-271.

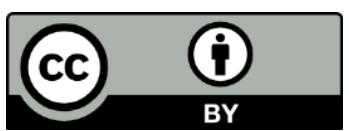

(C) 2020 Authors. Published by the University of Novi Sad, Faculty of Technical Sciences, Department of Graphic Engineering and Design. This article is an open access article distributed under the terms and conditions of the Creative Commons Attribution license 3.0 Serbia (http://creativecommons.org/licenses/by/3.0/rs/). 